\title{
Comparison of the SuperARMS and ARMS for detecting EGFR mutations in liquid- based cytology specimens from NSCLC patients
}

Wei Wu${ }^{\dagger}$, Ziyang Cao ${ }^{\dagger}$, Wei Zhang, Liping Zhang, Likun Hou and Chunyan Wu*

\begin{abstract}
Background: Non-surgical cytological specimens are adequate not only for accurate histological subtyping but also for molecular profiling. A modified amplification refractory mutation system polymerase chain reaction (ARMS PCR), known as SuperARMS PCR, was improved by optimizing the primers designation, which provides a higher sensitivity and specificity approach for free plasma DNA detection. It is unclear whether SuperARMS PCR detects epidermal growth factor receptor (EGFR) mutations in cytology samples. The aim of this study was to compare the EGFR mutations detected by ARMS PCR and SuperARMS PCR in cytology samples derived from advanced non-small cell lung cancer (NSCLC) patients.

Methods: From March 2016 to March 2018, a total of 234 cytological samples were obtained from primary or metastatic lesions of NSCLC, including 144 fine-needle aspirations (FNAs), 36 endobroncheal ultrasonography (EBUS) FNAs, 36 transbronchial needle aspirations (TBNAs) and 18 pleural effusion (PLEs). EGFR mutations were simultaneously detected using an ADx-ARMS EGFR kit (Amoy Diagnostics CO., Itd., Xiamen, China) and an ADx-SuperARMS EGFR kit (Amoy Diagnostics CO., Itd., Xiamen, China). Digital droplet PCR (ddPCR) and next-generation sequencing (NGS) were further used to verify the EGFR mutant inconsistent samples.

Results: All of the 234 patients with advanced or recurrent NSCLC were diagnosed and assessed by two cytopathologists, and their EGFR mutation statuses were successfully detected by ARMS and SuperARMS. Importantly, the SuperARMS and ARMS methods showed a highly concordant result of $94.0 \%$ (220/234) (95\%Cl: 85.0, 95.0\%). The positive rate of the SuperARMS was higher than the ARMS in the cytology samples for EGFR detection (46.2\% vs. 40.2\%). The specific EGFR mutation sites in 16 samples (6.8\%) were not completely consistent between the SuperARMS and ARMS. A total of 14 patients showed EGFR mutations when detected by SuperARMS, but by ARMS there were EGFR wild-type. Two patients were detected as having one more EGFR mutation site by SuperARMS than by ARMS. ddPCR and NGS were used to further confirm the EGFR mutations in these inconsistent samples. Eight samples had the same mutation results as the SuperARMS, and 6 samples were not verified because the remaining DNA was insufficient. A total of 78 EGFR mutation patients received Tyrosine Kinase Inhibitor (TKI) treatment. The overall objective response rate (ORR) was 88.5\% (69/78) for EGFR TKI treatment.
\end{abstract}

Conclusion: SuperARMS showed a high sensitivity and specificity for EGFR detection and thus, is expected to become a routine test in the clinic to be used as a widely available, easy-to-operate and sensitive method for EGFR mutation detection in liquid-based cytology samples.

Keywords: EGFR, SuperARMS, ARMS, NSCLC, Liquid-based cytology

\footnotetext{
* Correspondence: royzmy@outlook.com

${ }^{\dagger}$ Wei Wu and Ziyang Cao contributed equally to this work.

Department of Pathology, Shanghai Pulmonary Hospital, Tongji University

School of Medicine, 200433 Shanghai, People's Republic of China
}

(c) The Author(s). 2020 Open Access This article is distributed under the terms of the Creative Commons Attribution 4.0 International License (http://creativecommons.org/licenses/by/4.0/), which permits unrestricted use, distribution, and reproduction in any medium, provided you give appropriate credit to the original author(s) and the source, provide a link to the Creative Commons license, and indicate if changes were made. The Creative Commons Public Domain Dedication waiver (http://creativecommons.org/publicdomain/zero/1.0/) applies to the data made available in this article, unless otherwise stated. 


\section{Introduction}

Lung cancer remains the most common cause of cancer death worldwide. Approximately $70 \%$ of patients with nonsmall cell lung cancer (NSCLC) come to clinical attention at an advanced stage [1]. Small biopsy or cytological specimens may be a good choice in these patients, while they have no surgical options. The types of non-surgical cytological samples include fine-needle aspiration (FNA), endobronchial ultrasound-guided (EBUS) biopsy, transbronchial needle aspiration (TBNA), bronchoscopic brush (BB) and pleural effusion (PLE), which are reliable for the diagnosis and staging of thoracic malignancy, especially of NSCLC [2-6].

Many investigators report that cytological material obtained with minimally invasive procedures is adequate not only for accurate histological subtyping but also for molecular profiling [7-9]. In fact, studies have demonstrated optimal results using a multitude of cytological samples for molecular tests [10-12]. In our previous study, epidermal growth factor receptor (EGFR) mutation detection was performed by ARMS in liquid-based cytology samples from patients with NSCLC and their paired tissue samples, and the results of the two groups were identical [13]. Cytology specimens provide high quality DNA for the evaluation of clinically relevant mutations. Research groups, such as Allegrini $\mathrm{S}$ and Malapelle $\mathrm{U}$, have also directly used liquid-based cytology samples for EGFR and Kirsten rat sarcoma viral oncogene (KRAS) mutation detection with better results $[14,15]$. Under an era of individualized targeted therapy for NSCLC, it is crucial, for guiding treatment decisions, to obtain informative cytological material for both diagnosis and molecular testing. In our lab, we have increasingly used cytology specimens for molecular testing when cytology material was the only specimen source available.

Amplification Refractory Mutation System (ARMS) is an improved polymerase chain reaction (PCR) system and is an important platform for the detection of genes that drive NSCLC. The AmoyDx EGFR 29 Mutations Detection Kit is approved by the China Food and Drug Administration (CFDA) for the clinical testing of EGFR mutations using tissue samples. Recently, a novel technique called SuperARMS, which is a modified version of ARMS that optimizes the primers designation, was shown to provide a high sensitivity and specificity approach for free plasma DNA detection. No studies have been performed to compare ARMS and SuperARMS for EGFR mutation using cytology specimens. Therefore, it is unclear whether SuperARMS can be used to detect EGFR mutations in cytology samples and if it improves the sensitivity compared with ARMS. Thus, we conducted the present study to compare the EGFR mutations detected by ARMS and SuperARMS PCR in cytology samples derived from advanced NSCLC patients.

\section{Materials and methods}

\section{Cytology specimens and study design}

From March 2016 to March 2018, a total of 234 patients with advanced NSCLC were retrospectively enrolled in this study at the Shanghai Pulmonary Hospital, Tongji University. All the cytological samples were obtained from primary or metastatic lesions of NSCLC and included 144 FNAs, 36 EBUS FNAs, 36 TBNAs and 18 PLEs. The imaging data were independently reviewed by the authors to evaluate their treatment responses according to the Response Evaluation Criteria in Solid Tumors (RECIST) version 1.1. Progression-free survival (PFS) was calculated from the date of the initiating tyrosine kinase inhibitors (TKI) treatment to a radiologic or clinical observation of the disease progression.

A flowchart describing the study design is presented in Fig. 1. The study protocol was approved by the Institute Review Board of the Shanghai Pulmonary Hospital.

\section{Sample collection}

The liquid-based cytology specimen preparations were handled according to the standard specimen processing protocols in our laboratory. Cytology samples from FNAs, EBUS FNAs, TBNAs and PLEs were taken by clinicians and sent to pathology department for Thinprep cytologic test (TCT) within 1 day. One ThinPrep slide was stained with hematoxylin and eosin (H\&E) from the various cell types and was reviewed for morphologic evaluation by two cytopathologists. The remainder of the specimen liquidbased cytology specimens were rinsed in CytoLyt solution (Hologic) and were centrifuged to generate a cell pellet stored at $4{ }^{\circ} \mathrm{C}$. If the $\mathrm{H} \& \mathrm{E}$-stained smear was positive for NSCLC, we quantitated the number of tumor cells as more than 200 cells by the H\&E-stained smear and observed the residual cell pellet, which was visible to the naked eye, to ensure that the specimen was adequate for EGFR molecular testing.

\section{DNA extraction}

The residual cell pellets were used for the DNA extraction, which was performed using a Tissue DNA Kit (Amoy Diagnostics Co., Xiamen, China), following the manufacturer's instructions. The optical density of the extracted DNA samples was measured using a microplate spectrophotometer (Biotek). The A260/A280 value of all the samples was 1.8 to 2.1. The extracted DNA was used for EGFR molecular testing.

\section{EGFR mutation detection}

The EGFR mutations in the residual liquid-based cytology samples were simultaneously detected using an ADx-ARMS EGFR kitand an ADx-SuperARMS EGFR kit, according to the manufacturer's instructions. Briefly, the DNA templates were added to the EGFR Reaction 


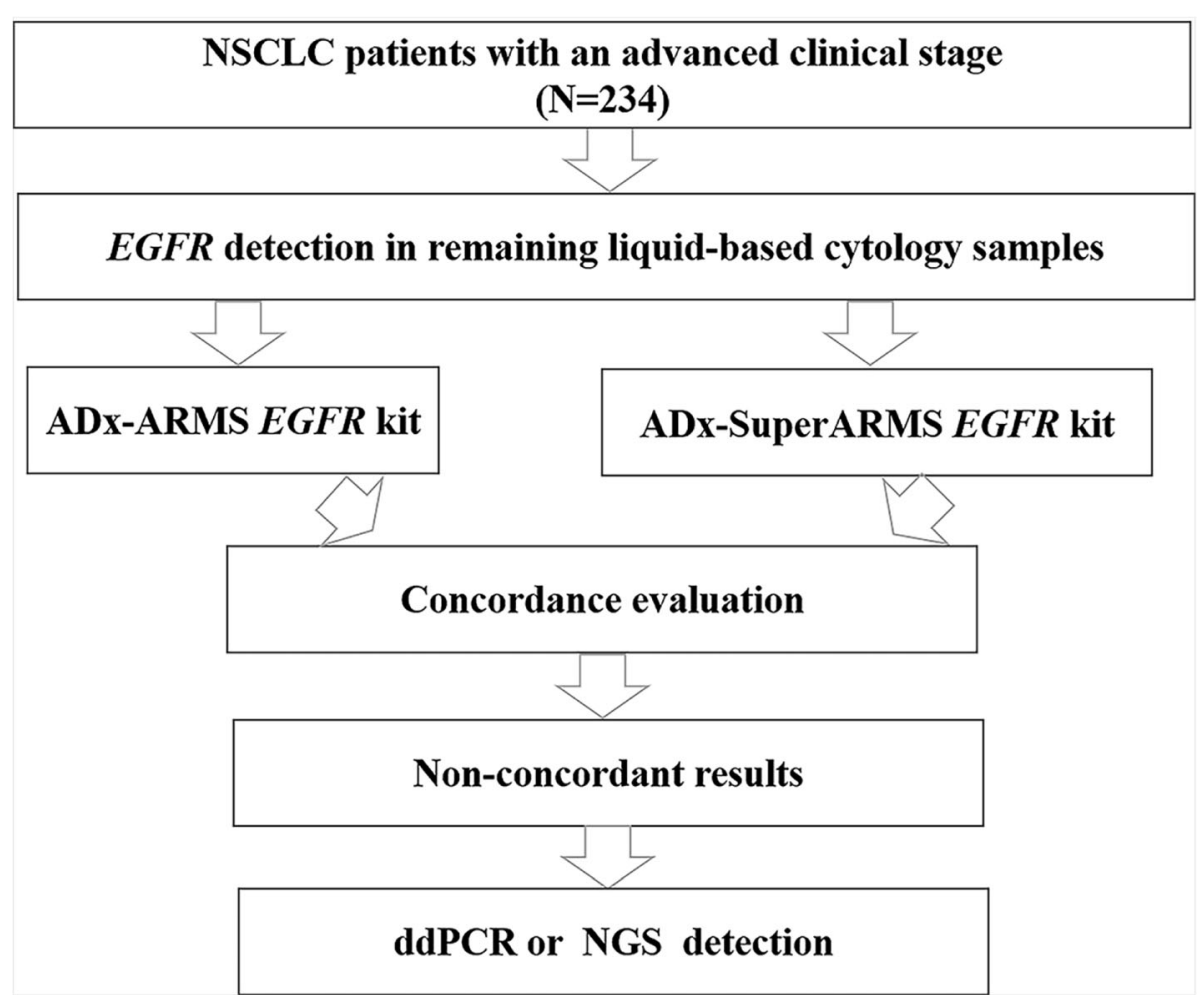

Fig. 1 A flowchart describing the study designAbbreviations: NSCLC = non-small cell lung cancer; EGFR = epidermal growth factor receptor; $\mathrm{NGS}=$ next-generation sequencing.

Mix, which included the primers, probes, dNTPs, buffer, $\mathrm{Mg} 2+$ and Taq DNA polymerases. The PCR reactions were performed on a Stratagene Mx3000P quantitative PCR (qPCR) system. After the PCR reaction was completed, the data interpretation was conducted according to results interpretation criteria of the EGFR mutation detection kit.

\section{ddPCR detection}

The ddPCR was performed according to the manufacturer's instructions. Briefly, the mutant reaction solution was prepared, and then, the emulsified microdroplets were generated in the QX200TM Droplet Generator instrument and were put into a 96-well plate for amplification. The PCR reaction conditions were as follows: incubation at $95^{\circ} \mathrm{C}$ for $10 \mathrm{~min}$, followed by 45 cycles of $95^{\circ} \mathrm{C}$ for $15 \mathrm{~s}$ and $60^{\circ} \mathrm{Cfor} 60 \mathrm{~s}$. After the PCR amplification, the 96-well plate was placed in the QX200 microdrop analyzer, and the data analyses were conducted using Quanta Soft analysis software.

\section{NGS detection}

DNA sequencing was carried out using a capture-based sequencing panel (Amoy Diagnostics CO., Ltd., Xiamen, China). The kit covered the targeted drug-related hot spot mutation regions of 10 genes, including EGFR et al. The liquid-based cytology DNA samples were sequenced with a NextSeq 500 (Illumina, Inc), with pair-end reads, with a reading length of PE150 and a sequencing depth of $>10,000 x$. The sequence data were analyzed using the AmoyDx NGS Data Analysis System ADXLC10 module (Amoy Diagnostics CO., Ltd., Xiamen, China).

\section{Statistical analysis}

The statistical analyses were performed with SPSS, version 17.0 (IBM, Armonk, NY). A two-sided tailed Fisher's exact test was applied to the data for one subgroup with a count less than 5. $p<0.05$ was considered significantly different.

\section{Results}

\section{Patient characteristics}

A total of 234 patients with advanced or recurrent NSCLC were retrospectively enrolled into the study. The patients' clinical characteristics are listed in Table 1 . The median age was 65 years (range, $31-85$ years). The patients consisted of 79 women and 155 men. Eighty-four patients were smokers, 145 were never-smokers, and 5 were unclear smokers. Fifty-eight patients were classified as at stage III, and 176 were at stage IV. The liquid-based cytology sample types included: FNA from the lungs for 114 patients, FNA from the lymph nodes for 30 patients, EBUS for 36 patients, TBNA for 36 patients and PLE for 15 patients. All the specimens were confirmed by two cytopathologists, 
Table 1 Clinical characteristics $(n=234)$

\begin{tabular}{ll}
\hline Factors & No.(\%) \\
\hline Sex & \\
Male & $155(48.7 \%)$ \\
Female & $79(48.7 \%)$ \\
Age, years & \\
Median & 65 \\
Range & $31-85$ \\
Smoking history & \\
Never & $145(62.0 \%)$ \\
Former/current & $84(35.9 \%)$ \\
Unclear & $5(2.1 \%)$ \\
Pathology & \\
Adenocarcinoma & $172(73.5 \%)$ \\
Squamous & $21(9.0 \%)$ \\
NSCLC & $41(17.5 \%)$ \\
Tumor Stage & \\
III & $58(24.8 \%)$ \\
IV & $176(75.2 \%)$ \\
Cytological sample types & \\
FNA (lung) & $114(48.7 \%)$ \\
FNA (lymph node) & $30(12.8 \%)$ \\
TBUS FNA & $36(15.4 \%)$ \\
Pleural effusion & $36(15.4 \%)$ \\
\hline
\end{tabular}

including 172 adenocarcinoma, 21 squamous and 41 NSCLC.

\section{Comparisons of the SuperARMS and the ADx-ARMS for detecting EGFR in the cytological specimens}

All 234 liquid-based cytology samples were successfully detected for their EGFR mutation status by ARMS and SuperARMS. The results are shown in Table 2. When tested by ARMS, 94 of 234 (40.2\%) patients were identified to have an EGFR mutation. When using the SuperARMS, 108 of 234 (46.2\%) patients were demonstrated to have an EGFR mutation. The positive rate of the SuperARMS was higher than the ARMS in the cytology samples for EGFR detection (46.2\% vs. $40.2 \%)$. The two-sided tailed Fisher's exact test showed that this was a significant difference $(p<0.05)$. The negative results and the positive results from the SuperARMS were in accordance with the ARMS results, at $90.0 \%$ (95\% CI, 91.0,97.1\%) and 100.0\% (94/94), respectively. The concordance rate of the EGFR mutation status between SuperARMS and ARMS was 94.0\% (220/ 234) (95\%CI:85.0,95.0\%). Fourteen patients showed an EGFR mutation by SuperARMS but were EGFR wild-type by ARMS. In addition, two patients were detected with
Table 2 Comparison of EGFR mutation status using ARMS and SuperARMS $(\mathrm{N}=234)$

\begin{tabular}{lllll}
\hline \multicolumn{4}{l}{ ARMS } & Total \\
\hline \multirow{3}{*}{ SuperARMS } & Mt & Wt & \\
& & 94 & 14 & 108 \\
& Wt & 0 & 126 & 126 \\
& Total & 94 & 140 & 234 \\
& PCR & $100 \%(94 / 94)$ & \\
& NCR* & $90 \%(126 / 140)(95 \% C l: 91.0,97.1 \%)$ \\
& Overall agreement* & $94 \%(220 / 234)(95 \% C l: 85.0,95.0 \%)$
\end{tabular}

*percentage [95\% confidence interval $(\mathrm{Cl})]$;

Mt: mutation; Wt: wild type; PCR: positive coincident rate; NPV: negative coincident rate

one more EGFR mutation site by SuperARMS than by ARMS.

SuperARMS for detecting specific EGFR mutation types Out of total of 234 samples, 16 cases were discordant between SuperARMS and ARMS (Table 3). Among them, 14 (87.5\%) cases were detected as positive for EGFR mutations only by SuperARMS, while the other two samples showed different mutations by these two methods. We performed ddPCR and NGS to further confirmed the inconsistent $\operatorname{samples}(n=12 / 16,4$ samples were insufficient). 1 sample with EGFR 20-ins mutation (Sample ID:FK020) is beyond capability of ddPCR, so $11 / 12$ samples were identified by ddPCR at first. Then we successful detected 5 sample using NGS (Table 3). 2 samples were negative for mutations。 2 samples had a special L858R mutation type (NM_005228.3: exon21:c.2573 T > G:p.L858R), which was only detected by NGS and SuperARMS. This observation indicates that SuperARMS and NGS methods can detect more sites of mutation than ARMS in the tested regions.

EGFR mutation status and prediction of EGFR-TKI efficacy As shown in Table 4, out of the 94 positive EGFR mutation patients by ARMS, 77 patients received EGFR-TKI treatment. In another 14 patients with an EGFR mutation by SuperARMS, only 1 patient received EGFR-TKI treatment. The ORR was $88.5 \%$ (69/78) for EGFR TKI treatment. In patients with or without EGFR mutation, 81 received chemotherapy. The median PFS was significantly prolonged in the EGFR mutation patients compared with the EGFR wild-type patients (18.0 months vs 8.3 months, $P<0.01$ ) (Fig. 2). These data suggested that EGFR mutation status in cytology samples detected by ARMS and SuperARMS is predictive of tumor response to EGFR-TKIs and survival results.

\section{Discussion}

Currently, the molecular spectrum of tumor patients promotes the development of individualized treatment. 
Table 3 Sixteen Nonconcordant Cases of SuperARMS and ARMS for Cytology EGFR Detection

\begin{tabular}{|c|c|c|c|c|}
\hline \multicolumn{2}{|c|}{ Sample ID } & \multirow{2}{*}{$\frac{\text { ARMS }}{19-\mathrm{DEL} / \mathrm{T} 790 \mathrm{M}}$} & \multirow{2}{*}{$\begin{array}{l}\text { Super-ARMS } \\
\text { 19-DEL/T790 M }\end{array}$} & \multirow{2}{*}{$\begin{array}{l}\mathrm{ddPCR} \\
\mathrm{NA}\end{array}$} \\
\hline FK008 & 19-DEL & & & \\
\hline FK020 & WT & 20-INS & NA & 20-INS \\
\hline FK055 & WT & L858R & WT & NE DNA \\
\hline FK062 & WT & L858R & WT & NM_005228.3:exon21:c.2573_2574delinsGT:p.L858R \\
\hline FK066 & WT & L858R & WT & NM_005228.3:exon21:c.2573 T > G:p.L858R \\
\hline FK080 & WT & 19-DEL & 19-DEL & NA \\
\hline FK108 & WT & L858R & L858R & NA \\
\hline FK129 & WT & L858R & NE DNA & NE DNA \\
\hline FK130 & WT & L858R & NE DNA & NE DNA \\
\hline FK135 & WT & 19-DEL & 19-DEL & NA \\
\hline FK147 & WT & 19-DEL & WT & WT \\
\hline FK166 & WT & L858R & WT & NE DNA \\
\hline FK190 & WT & 19-DEL & WT & WT \\
\hline FK206 & 19-DEL & 19-DEL/L858R & NE DNA & NE DNA \\
\hline FK220 & WT & 20-ins & NE DNA & NE DNA \\
\hline FK221 & WT & 19-DEL/L858R & 19-DEL/L858R & NA \\
\hline
\end{tabular}

*NE DNA: not enough DNA

Especially in patients with NSCLC, the mutation status of EGFR has great guiding significance for the use of TKIs [16]. Now more than ever, obtaining adequate material for the molecular testing of NSCLC is crucial. In the clinical setting, a cytological specimen may be the only material available for the determination of EGFR mutation status. Liquid-based cytology specimens have been used for molecular assays with optimal results, although rare large series have been published.

It has been reported that the number of tumor cells in the remaining liquid base cell sample varies significantly between the different samples, with limited sensitivity of the ARMS detection in low tumor cell content samples [17]. SuperARMS is a highly sensitive kit that detects 41 EGFR mutations in exons 18 to 21 in human plasma cfDNA samples. To the best of our knowledge, ours is the first study to evaluate the performance of SuperARMS in liquid-based cytology samples for EGFR detection.

The feasibility of conventional smears and the remaining liquid-based cytology samples for molecular detection has been evaluated in multiple laboratories [1820]. Gilda da Cunha Santos's team compared the nucleic acid quality of the remaining liquid-based cytology samples, smears and cell blocks for molecular detection, and found that well-preserved liquid-based cytology samples had a good nucleic acid yield and quality [21]. Maurizio Martini's research team explained the role of cytological samples in tumor diagnosis, which is not only used in the diagnosis, but also in molecular testing, and a small amount of high-quality nucleic acid provides effective molecular genetic features [22]. Consistent with these reports in the literature, our liquid-based cytology samples had good DNA quality and or the EGFR gene status was successfully detected using SuperARMS and ARMS.

In this study, various cytological specimens, including FNA, EBUS FNA, TBNA and PLE, were all subjected to the EGFR mutational analysis using SuperARMS and ARMS, and $100 \%(234 / 234)$ of the samples were suitable for testing. Firstly, the SuperARMS and ARMS methods showed highly concordant results, at 94.0\% (220/234) (95\%CI: 85.0, 95.0\%). Moreover, SuperARMS detected more mutations compared with ARMS. Our study showed that SuperARMS, as a highly sensitive molecular detection method, can be used for the detection of EGFR not only in plasma ctDNA, but also in cytological samples DNA.

The high sensitivity and specificity of SuperARMS has been demonstrated in a series of liquid biopsy studies $[23,24]$. Our study confirmed that SuperARMS had high

Table 4 EGFR Mutation Status and TKIs treatment

\begin{tabular}{llllll}
\hline & Sample No. & Positive No. & Negtive No. & Sensitive MT No. & targeted therapy No. \\
\hline ARMS & 234 & 94 & 140 & 92 & 77 \\
SuperARMS & 234 & 108 & 126 & 104 & 78 \\
\hline
\end{tabular}




\section{Progression Free Survival Functions}

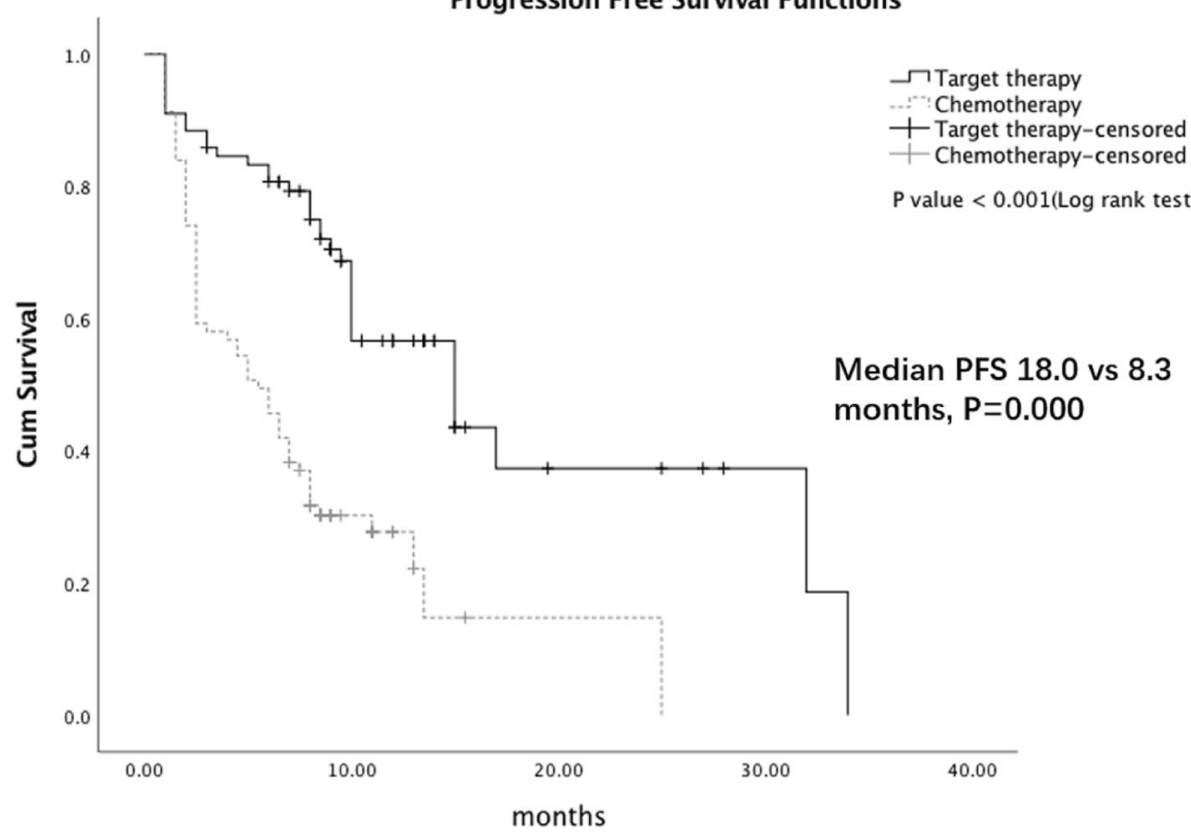

Fig. 2 PFS in EGFR mutation patients compared with EGFR wild-type patients

sensitivity and specificity in liquid-based cytology samples. The positive rate of SuperARMS was higher than ARMS in the cytology samples for EGFR detection (46.2\% vs. $40.2 \%$ ) (Table 2). Our study showed that SuperARMS was not only used in the blood, but also in liquid-based cytology samples. Is there a false positive result in the SuperARMS? We analyzed the reasons for the inconsistency of the results of the validation samples. The false positive results caused by the contamination of aerosol in the laboratory should be excluded. Notably that two samples had the special L858R mutation type (NM_005228.3: exon21:c.2573 T > G:p.L858R), which was detected by SuperARMS and NGS. The specific reason for this observation is that the SuperARMS and NGS methods detect more sites.

Many previous studies report that there is a significant correlation between EGFR mutation status and treatment for PFS $[25,26]$. Consistent with these studies, our study revealed that patients with EGFR mutations, using targeted drugs as the first line of treatment, had a longer PFS compared to patients without EGFR mutations, using chemotherapy drugs as the first line of treatment, as detected by both ARMS and SuperARMS.

In conclusion, the SuperARMS had a high sensitivity and specificity for EGFR detection in liquid-based cytology samples, and these results were good predictors of the efficacy of EGFR-TKIs. Thus, the SuperARMS is expected to become a test that is used routinely in the clinic as a widely available, easy-to-operate and sensitive method for EGFR mutation detection in liquid-based cytology samples.

\section{Abbreviations}

20-ins: 20 exon insertion mutation; ARMS: amplification Refractory Mutation System; BB: bronchoscopic brush; EBUS: endobronchial ultrasound-guided;

EGFR: epidermal growth factor receptor; FNA: fine-needle aspiration; H\&E: hematoxylin and eosin; NSCLC: non-small cell lung cancer;

ORR: objective response rate; PCR: polymerase chain reaction;

PFS: progression-free survival; PLE: pleural effusion; RECIST: response

Evaluation Criteria in Solid Tumors; TBNA: transbronchial needle aspiration;

TKI: tyrosine kinase inhibitor

\section{Acknowledgements}

We thank all laboratory members in the Department of Pathology, Shanghai Pulmonary Hospital, for their kind help.

\section{Ethical approval and consent to participate}

The study was approved by the ethics committee of Shanghai Pulmonary Hospital (No. K17-040). Additional patient consent for this retrospective

study was not required.

\section{Authors' contributions}

WW, ZY C, LK H and CY W designed the study. WW, ZY C, LP Z and WZ analyzed and interpreted the patient data. $W W, Z Y C$ and $Z W$ performed the experiments. All authors read and approved the final manuscript.

\section{Funding}

This study was sponsored by "Shanghai Sailing Program" (17YF1415800).

Availability of data and materials

The raw data are available upon request.

\section{Consent for publication}

Not applicable.

\section{Competing interests}

The authors declare that they have no competing interests.

Competing interests

The authors declare that they have no competing interests. 
Received: 27 September 2019 Accepted: 26 November 2019

Published online: 31 January 2020

\section{References}

1. Walker S. Updates in non-small cell lung cancer. Clin J Oncol Nurs. 2008; 12(4):587-96.

2. Fielding D, Kurimoto N. Endobronchial ultrasound-guided transbronchia needle. Aspiration for diagnosis and staging of lung cancer. Clin Chest Med. 2018:39(1):111-23.

3. Roh MH. The utilization of cytologic fine-needle aspirates of lung cancer for molecular diagnostic testing. J Pathol Transl Med. 2015:49(4):300-9.

4. Arias S, Liu QH, Frimpong B, et al. Role of the Endobronchial landmarks guiding TBNA and EBUS-TBNA in lung Cancer staging. Can Respir J. 2016; 2016:1652178.

5. Steinfort DP, Leong TL, Laska IF, et al. Diagnostic utility and accuracy of rapid on-site evaluation of bronchoscopic brushings. Eur Respir J. 2015;45(6): 1653-60.

6. Lococo F, Cesario A, Attili F, Chiappetta M, et al. Transoesophageal endoscopic ultrasound-guided fine-needle aspiration of pleural effusion for the staging of non-small cell lung cancer. Interact Cardiovasc Thorac Surg. 2013;17(2):237-41.

7. Roy-Chowdhuri S, Aisner DL, Allen TC, et al. Biomarker testing in lung carcinoma cytology specimens: a perspective from members of the pulmonary pathology society. Arch Pathol Lab Med. 2016:140(11):1267-72.

8. Ellison G, Zhu G, Moulis A, et al. EGFR mutation testing in lung cancer: a review of available methods and their use for analysis of tumour tissue and cytology samples. J Clin Pathol. 2013;66(2):79-89.

9. Wei S, Lieberman D, Morrissette JJ, et al. Using "residual" FNA rinse and body fluid specimens for next-generation sequencing: an institutional experience. Cancer Cytopathol. 2016;124:324-9.

10. Treece AL, Montgomery ND, Patel NM, et al. FNA smears as a potential source of DNA for targeted next-generation sequencing of lung adenocarcinomas. Cancer Cytopathol. 2016;124:406-14.

11. da Cunha Santos G, Saieg MA. Preanalytic specimen triage: smears, cell blocks, cytospin preparations, transport media, and cytobanking. Cancer Cytopathol. 2017;125(S6):455-64.

12. Roy-Chowdhuri S, Chen $\mathrm{H}$, Singh RR, et al. Concurrent fine needle aspirations and core needle biopsies: a comparative study of substrates for nextgeneration sequencing in solid organ malignancies. Mod Pathol. 2017; 30(4):499-508

13. Wu CY, Hou LK, Ren SX, et al. High feasibility of liquid-based cytological samples for detection of EGFR mutations in Chinese patients with NSCLC. Asian Pac J Cancer Prev. 2014;15:7885-9.

14. Allegrini S, Antona J, Mezzapelle R, et al. Epidermal growth factor receptor gene analysis with a highly sensitive molecular assay in routine cytologic specimens of lung adenocarcinoma. Am J Clin Pathol. 2012;138(3):377-81.

15. Malapelle $U$, de Rosa N, Rocco D, et al. EGFR and KRAS mutations detection on lung cancer liquid-based cytology: a pilot study. J Clin Pathol. 2012;65(1): 87-91.12.

16. Juan $\mathrm{O}$, Popat $\mathrm{S}$. Treatment choice in epidermal growth factor receptor mutation-positive non-small cell lung carcinoma: latest evidence and clinical implications. Ther Adv Med Oncol. 2017:9(3):201-16.

17. Roy-Chowdhuri S, Aisner DL, Allen TC, et al. Biomarker testing in lung carcinoma cytology specimens. Arch Pathol Lab Med. 2016;140:1267-72.

18. Betz BL, Roh MH, Weigelin HC, et al. The application of molecular diagnostic studies interrogating EGFR and KRAS mutations to stained cytologic smears of lung carcinoma. Am J Clin Pathol. 2011;136(4):564-71.

19. Reynolds JP, Zhou Y, Jakubowski MA, et al. Next-generation sequencing of liquid-based cytology non-small cell lung cancer samples. Cancer Cytopathol. 2017;125(3):178-87.

20. Doxtader EE, Cheng YW, Zhang Y. Molecular testing of non-small cell lung carcinoma diagnosed by Endobronchial ultrasound-guided Transbronchial fine-needle aspiration: the Cleveland Clinic experience. Arch Pathol Lab Med. 2019;143(6):670-6.

21. da Cunha Santos G, Saieg MA. Preanalytic specimen triage: Smears, cell blocks, cytospin preparations, transport media, and cytobanking. Cancer Cytopathol. 2017;125(S6):455-64.

22. Martini M, Capodimonti S, Cenci T, et al. To obtain more with less: Cytologic samples with ancillary molecular techniques-the useful role of liquid-based cytology. Arch Pathol Lab Med. 2018;142(3):299-307.
23. Cui $\mathrm{S}$, Ye $\mathrm{L}$, Wang $\mathrm{H}$, et al. Use of SuperARMS EGFR mutation detection kit to detect EGFR in plasma cell-free DNA of patients with lung adenocarcinoma. Clin Lung Cancer. 2018;19(3):e313-22.

24. Feng WN, Gu WQ, Zhao N, et al. Comparison of the SuperARMS and droplet digital PCR for detecting EGFR mutation in ctDNA from NSCLC patients. Transl Oncol. 2018;11(2):542-5.

25. $\mathrm{Xu} \mathrm{J,} \mathrm{Jin} \mathrm{B,} \mathrm{Chu} \mathrm{T,} \mathrm{et} \mathrm{al.} \mathrm{EGFR} \mathrm{tyrosine} \mathrm{kinase} \mathrm{inhibitor} \mathrm{(TKI)} \mathrm{in} \mathrm{patients} \mathrm{with}$ advanced non-small cell lung cancer (NSCLC) harboring uncommon EGFR mutations: a real-world study in China. Lung Cancer. 2016:96:87-92.

26. Sutiman N, Tan SW, Tan EH, et al. EGFR mutation subtypes influence survival outcomes following first-line Gefitinib therapy in advanced Asian NSCLC patients. J Thorac Oncol. 2017;12(3):529-38.

\section{Publisher's Note}

Springer Nature remains neutral with regard to jurisdictional claims in published maps and institutional affiliations.
Ready to submit your research? Choose BMC and benefit from:

- fast, convenient online submission

- thorough peer review by experienced researchers in your field

- rapid publication on acceptance

- support for research data, including large and complex data types

- gold Open Access which fosters wider collaboration and increased citations

- maximum visibility for your research: over $100 \mathrm{M}$ website views per year

At BMC, research is always in progress.

Learn more biomedcentral.com/submissions 